IASSNS-HEP-00/25

CERN-TH/2000-108

EFI-2000-17

hep-th/0005276

\title{
Saltatory Relaxation of the Cosmological Constant t $^{1}$
}

\author{
Jonathan L. Feng*, John March-Russell ${ }^{\dagger}$, Savdeep Sethi* ${ }^{*}$ \\ and Frank Wilczek* \\ * School of Natural Sciences, Institute for Advanced Study \\ Princeton, NJ 08540 USA \\ $\dagger$ Theory Division, CERN, CH-1211, Geneva 23, Switzerland \\ $\ddagger$ Enrico Fermi Institute, University of Chicago \\ Chicago, IL 60637 USA
}

\begin{abstract}
We modify and extend an earlier proposal by Brown and Teitelboim to relax the effective cosmological term by nucleation of branes coupled to a three-index gauge potential. Microscopic considerations from string/M theory suggest two major innovations in the framework. First, the dependence of brane properties on the compactification of extra dimensions may generate a very small quantized unit for jumps in the effective cosmological term. Second, internal degrees of freedom for multiply coincident branes may enhance tunneling rates by exponentially large density of states factors. These new features essentially alter the relaxation dynamics. By requiring stability on the scale of the lifetime of the universe, rather than absolute stability, we derive a non-trivial relation between the supersymmetry breaking scale and the value of the cosmological term. It is plausibly, though not certainly, satisfied in Nature.
\end{abstract}

\footnotetext{
${ }^{1}$ feng@ias.edu, jmr@nxth04.cern.ch, sethi@theory.uchicago.edu,wilczek@ias.edu
} 


\section{Introduction}

Although both the Casimir effect of quantum theory and the existence of symmetrybreaking condensates in both the strong and the electroweak sectors of the Standard Model indicate that empty space is a dynamical medium that 'ought to' have a large mass density, gravity, which couples universally to mass, does not reveal it. This is the problem of the cosmological term [1]. Various mechanisms have been proposed to address this problem, but so far none has won wide acceptance. This situation is especially challenging for string theory, and its conjectured non-perturbative definition $\mathrm{M}$ theory, in as much as string theory is proposed as a fully specified dynamical theory of gravity.

An interesting approach to the solution of the cosmological term problem is the proposal that it is relaxed by jumps (saltation) associated with some rather exotic dynamics. There is an important conceptual advantage to having the relaxation connected to some - necessarily exotic — dynamics that responds only to a source taking the form of an effective cosmological term. For if the dynamics responds to several influences, it is difficult to see how a particularly simple value for one partial determinant of its behavior can become overwhelmingly preferred. A model for this logic is the advantage one gains from Peccei-Quinn symmetry in relieving the $\theta$-problem of QCD. Indeed, that symmetry produces an exotic dynamics (the axion field) which responds only to a source having the form of an effective $\theta$ term. Note that this logic also applies, in connection with continuous relaxation of scalar fields, to self-interactions (including kinetic terms), as has been quantified by Weinberg [1].

Saltation in the effective cosmological term has been considered in the context of stepwise false vacuum decay [2, 3] in a quasi-periodic staircase potential by Abbott [4], or alternatively by Brown and Teitelboim (BT) through nucleation of fundamental membrane degrees of freedom [5]. Since the membrane formulation is close in spirit to the ones that appear to arise naturally in string theory, we shall phrase our discussion in its framework.

The essential ingredients in the BT model are a fundamental membrane degree of freedom (in modern language, a 2-brane) and a 4-form gauge field strength $F_{4}$ (deriving from a 3 -form potential). The world-volumes of the membranes couple to the 3 -form potential. The field strength $F_{4}$ has no other couplings and no local dynamics in the four-dimensional spacetime, but its expectation value contributes to the effective cosmological term. The presence of an expectation value for $F_{4}$ induces the nucleation of the 2-brane to which it couples, in a manner analogous to the Schwinger mechanism for electric field decay through nucleation of $e^{+} e^{-}$pairs. When a membrane is nucleated, say as a spherical shell, the effective value of the cosmological term on the inside differs from its previous value (the value on the outside) by an amount proportional to the coupling constant of the membrane. If a membrane of the correct sign is nucleated, the contribution of the 4-form to the effective value of the cosmological term will be reduced. For a suitable value of $\left\langle F_{4}\right\rangle$, this contribution can 
cancel that arising from the combined effects of all other degrees of freedom in the theory. If the steps between adjacent false vacua are sufficiently small, and if there is a reason why vacuum decay stops or slows down dramatically as $\Lambda_{\text {eff }} \rightarrow 0$, this mechanism could in principle relax a large microscopic cosmological term (arising from all sources other than the membrane- $F$ field dynamics) to a value within observational bounds.

In their pioneering work, Abbott, and Brown and Teitelboim, postulated dynamical entities ad hoc, with no richer context to enhance their credibility or connect them to other problems and facts of physics. On the other hand, developments in string theory in the past few years have emphasized the importance of extended objects branes - of various types. It therefore seems appropriate to consider whether these branes might allow improved mechanisms for saltatory relaxation of the cosmological term. We believe that this is the case, for several reasons:

- A wide variety of membranes play a fundamental role in string theory. There are corresponding gauge fields, which naturally couple (only) to these extended objects.

- Since the theory is naturally formulated in higher dimensions, the couplings of these membranes as seen in four dimensions are not fixed and quantized, but rather are determined in terms of the fundamental (fixed, quantized) couplings together with properties of the extra-dimensional compactification.

- Similarly, the effective tension as seen in four dimensions depends on the properties of the extra-dimensional compactification.

- There are significant and, in suitable cases, exponentially large, density of states factors associated with semi-classical brane processes.

- Small tension, which may be favored for dynamical reasons, and large density of states factors make possible rapid relaxation of the cosmological constant.

We should stress that regardless of whether membrane nucleation is the final solution, or perhaps an ingredient, in solving the riddle of the cosmological constant, nucleation processes involving extended objects generically occur in string theory. Studying these processes is likely to give some insight into the question of vacuum selection and into the question of a background independent formulation of string theory.

In the following section, we review the BT formalism for relaxation of the cosmological constant through brane nucleation and the primary obstacles encountered. In the next two sections, we discuss features of string theory that may alleviate these difficulties: in Sec. 目 we describe the possibility of small charge densities and tensions arising from compactification, and in Sec. 6 we note the relevance of exponentially large density of states factors. Motivated by these features, we describe two possible 
scenarios for the cosmological constant in Sec. 5. In Sec. 6, we discuss a number of outstanding issues and summarize.

\section{Relaxation Dynamics}

\subsection{Basic mechanism}

We now recall the basic dynamics of the BT mechanism [5], which we have been able to express in a somewhat simplified fashion. For other recent discussions of membrane nucleation, see 6].

Consider gravity in $D=4$ spacetime dimensions with a 2-brane $X^{\alpha}$ coupled to a 3 -form gauge potential $A_{3}$. The Minkowski action is

$$
\begin{aligned}
S_{M}= & -\tau_{2} \int d^{3} \xi \sqrt{-\operatorname{det} g_{a b}}+\frac{\rho_{2}}{6} \int d^{3} \xi A_{\alpha \beta \gamma} \frac{\partial X^{\alpha}}{\partial \xi^{a}} \frac{\partial X^{\beta}}{\partial \xi^{b}} \frac{\partial X^{\gamma}}{\partial \xi^{c}} \varepsilon^{a b c} \\
& -\frac{1}{48} \int d^{4} x \sqrt{-g} F_{\alpha \beta \gamma \delta} F^{\alpha \beta \gamma \delta}+\frac{1}{6} \int d^{4} x \partial_{\alpha}\left[\sqrt{-g} F^{\alpha \beta \gamma \delta} A_{\beta \gamma \delta}\right] \\
& +\frac{1}{2} \int d^{4} x \sqrt{-g} M^{2}(R-2 \lambda)-M^{2} \oint d^{3} x \sqrt{h} K
\end{aligned}
$$

where the $\xi^{a}$ parameterize the membrane world-volume, and $g_{a b}=\partial_{a} X^{\alpha} \partial_{b} X_{\alpha}$ is the induced world-volume metric. The surface integral is over spacetime boundaries with $h$ and $K$ the induced metric and extrinsic curvature, respectively. This term and the total derivative integral ensure that the action has well-defined functional derivatives with respect to the metric and gauge field. An important point is that in four dimensions the 4-form field strength contains no independent propagating degrees of freedom, its value, up to a constant, being fully determined by the background of sources charged with respect to $A_{\alpha \beta \gamma}$.

The parameters entering this action, and their mass dimensions in $D=4$, are

$\begin{array}{lcl}\text { 2-brane tension } & \tau_{2} & 3 \\ \text { 2-brane charge density } & \rho_{2} & 2 \\ \text { (Reduced) Planck mass } & M & 1 \\ \text { Bare cosmological constant } & \lambda & 2 .\end{array}$

Numerically, $M=(8 \pi G)^{-1 / 2}=2.4 \times 10^{18} \mathrm{GeV}$. Here, in agreement with BT, we use the canonical - positive energy - sign for the $F^{2}$ term. In Sec. 3, we shall see that this is appropriate for the branes relevant to us.

Rotating to Euclidean space, we find

$$
\begin{aligned}
S_{E}= & \tau_{2} \int d^{3} \xi \sqrt{\operatorname{det} g_{a b}}+\frac{\rho_{2}}{6} \int d^{3} \xi A_{\alpha \beta \gamma} \frac{\partial X^{\alpha}}{\partial \xi^{a}} \frac{\partial X^{\beta}}{\partial \xi^{b}} \frac{\partial X^{\gamma}}{\partial \xi^{c}} \varepsilon^{a b c} \\
& -\frac{1}{48} \int d^{4} x \sqrt{g} F_{\alpha \beta \gamma \delta} F^{\alpha \beta \gamma \delta}+\frac{1}{6} \int d^{4} x \partial_{\alpha}\left[\sqrt{g} F^{\alpha \beta \gamma \delta} A_{\beta \gamma \delta}\right] \\
& +\int d^{4} x \sqrt{g} \frac{1}{2} M^{2}(-R+2 \lambda)+M^{2} \oint d^{3} x \sqrt{h} K .
\end{aligned}
$$


The sign of the $F^{2}$ term in Eq. (3) depends on the Euclideanization procedure. Here, following Ref. [5], we make the conventional rotations $x^{0} \rightarrow-i x^{0}, X^{0} \rightarrow-i X^{0}$ for time-like quantities, but take $A^{0 \mu_{2} \ldots \mu_{D-1}} \rightarrow A^{0 \mu_{2} \ldots \mu_{D-1}}$ and $A^{\mu_{1} \ldots \mu_{D-1}} \rightarrow i A^{\mu_{1} \ldots \mu_{D-1}}$ for the space-like components so that the field strength $F$ is invariant. Alternatively, one may adopt the prescription $A^{0 \mu_{2} \ldots \mu_{D-1}} \rightarrow-i A^{0 \mu_{2} \ldots \mu_{D-1}}$ and keep the space-like components invariant. In this case, the sign of the $F^{2}$ term in Eq. (3) changes. However, the field strength is not invariant under this prescription; taking $\left\langle F_{4}\right\rangle$ in Eq. (3) to be pure imaginary [7] leaves the following analysis unchanged.

The instanton solution is a membrane that divides space into two regions, an outside $O$ and an inside $I$. In each region, the field strength is a constant

$$
\left\langle F_{O, I}^{\alpha \beta \gamma \delta}\right\rangle=\frac{c_{O, I}}{\sqrt{g}} \varepsilon^{\alpha \beta \gamma \delta},
$$

and the field strengths are matched across the membrane boundary via

$$
c_{I}=c_{O}-\rho_{2} .
$$

The effective cosmological terms are

$$
\Lambda_{O, I}=\lambda+\frac{1}{2 M^{2}} c_{O, I}^{2},
$$

where the field strength contribution follows from Einstein's equations. Alternatively, it may be 'read off' from the action if one is careful to include the on-shell contribution from the total derivative term, which is double in magnitude and opposite in sign relative to the usual $F^{2}$ term. From Eq. (6), it is clear that if the bare cosmological term is to be canceled, it must be negative, and we therefore assume $\lambda<0$.

The tunneling probability is $P \sim e^{-B}$, where the bounce action [2, 3] for this false vacuum decay is

$$
B= \begin{cases}\infty, & \text { if } \Lambda_{\mathrm{O}}, \alpha_{\mathrm{O}}<0 \\ 12 \pi^{2} M^{2}\left[\frac{1}{\Lambda_{O}}\left(1-b \alpha_{O}\right)-\frac{1}{\Lambda_{I}}\left(1-b \alpha_{I}\right)\right], & \text { otherwise }\end{cases}
$$

Here the bubble radius, defined so that the area of the bubble slice when continued back to Minkowski signature is $4 \pi b^{2}$, is

$$
b=\frac{1}{\sqrt{\frac{1}{3} \Lambda_{O}+\alpha_{O}^{2}}}=\frac{1}{\sqrt{\frac{1}{3} \Lambda_{I}+\alpha_{I}^{2}}},
$$

and

$$
\begin{aligned}
\alpha_{I} & =\frac{1}{3 x M}\left[c_{O}-\left(\frac{1}{2} \pm \frac{3}{4} x^{2}\right) \rho_{2}\right] \\
\Lambda_{I} & =\Lambda_{O}+\frac{1}{2 M^{2}}\left(\rho_{2}^{2}-2 \rho_{2} c_{O}\right) \\
x & =\frac{\tau_{2}}{\rho_{2} M} .
\end{aligned}
$$

In the following sections, we will often work in Planck units with $M \equiv 1$. 


\subsection{Naturalness}

The value of the cosmological term at present is

$$
\Lambda_{\mathrm{obs}} M^{2} \lesssim\left(2 \times 10^{-3} \mathrm{eV}\right)^{4} \sim 10^{-120} M^{4}
$$

Present data prefer a non-zero, positive value; for recent reviews, see [8]. One virtue of saltatory relaxation is that, in contrast to mechanisms involving symmetries or continuous relaxation, a small non-zero value may emerge naturally as a consequence of a non-vanishing jump size.

For the observed value to be natural in the framework of brane nucleation, the spacing between allowed values of the effective cosmological term, near the observed value, cannot be much larger. This translates into the condition

$$
\rho_{2} \lesssim \frac{\Lambda_{\mathrm{obs}}}{|\lambda|^{1 / 2}}
$$

This is an extremely stringent condition on the microphysics, even for plausible $|\lambda| \ll$ 1 , since the observed cosmological constant is so small. For example, even if the bare cosmological constant is generated only at the $\mathrm{TeV}$ scale through low-energy supersymmetry breaking so that $|\lambda| \sim 10^{-60}$, one still requires $\rho_{2} \lesssim 10^{-90}$, which translates into an associated mass scale of $10^{-18} \mathrm{eV}$. The requirement of such a small coupling is, at best, unsettling, and one might hope for an explanation in some more fundamental framework. This is especially true in string theory, where, in the absence of such an explanation, one naively expects couplings to be of order the Planck scale.

As an aside, note that throughout this study, we consider evolution of the cosmological term down the staircase of values allowed by brane nucleation with fixed charge density $\rho_{2}$, and thus with essentially fixed step size. However, very small fractional changes in $\rho_{2}$, of order

$$
\frac{\delta \rho_{2}}{\rho_{2}} \lesssim \frac{\Lambda_{\mathrm{obs}}}{|\lambda|}
$$

assuming $\rho_{2} \ll \sqrt{|\lambda|}$, are sufficient to bring the observed value of the effective cosmological term into range by distorting the size of the steps near $\Lambda=0$. Another possibility is that the entire staircase moves up or down by some suitably small amount. Although we will not attempt to exploit these features here, they may play an important role in future work, since, as we shall emphasize below, $\rho_{2}$ is in principle a dynamical variable. It depends, in particular, on the expectation values of the string theory compactification moduli, including the dilaton.

\subsection{Absolute Stability}

The cosmological term must not only relax to within its observational bounds, but it must also stop evolving once it reaches this interval. For de Sitter space, additional bubble nucleations are always possible. However, for $\Lambda_{O}<0$, transitions can take 
place only when $\alpha_{O}>0$. It is not hard to show that this constraint, along with the condition $\frac{1}{3} \Lambda_{O}+\alpha_{O}^{2}>0$, implies that further lowering of the effective cosmological term will not occur beyond the first anti-de Sitter step if the tension is sufficiently large. This remarkable result is closely related to the Coleman-de Luccia [3] gravitational suppression of false vacuum decay. BT hypothesized that our universe is at the endpoint of such an evolution.

A sufficient condition to ensure absolute vacuum stability is $\tau_{2}^{2}>\frac{4}{3} \rho_{2} c_{O}$ or, in terms of the tension to charge density ratio,

$$
x=\frac{\tau_{2}}{\rho_{2}} \gtrsim \sqrt{\frac{|\lambda|}{\Lambda_{\mathrm{obs}}}} .
$$

Thus, even for the smallest plausible $|\lambda|$, a large hierarchy between tension and charge density is required.

More problematic still, BT showed that, upon combining the stability and naturalness conditions, the time required to reach the endpoint is excessively large, so large that even the very slow inflation that occurs in the penultimate vacuum would leave the universe entirely devoid of matter and energy.

\section{Saltation in String Theory: Tension and Charge Density}

\subsection{Framework}

In its long wavelength approximation, M theory supports BPS M2-branes and M5branes [9, 10, 11]. The M2-branes couple electrically to the 3-index gauge potential of 11-dimensional supergravity, which we denote by $A_{3}$. We define a dual gauge potential $A_{6}$ in the standard way:

$$
F=d A_{3}=* F_{7}=* d A_{6}
$$

The M5-branes couple magnetically to $A_{3}$, or directly to $A_{6}$. In terms of the 11dimensional Planck scale $l_{p}$, M2-branes have a tension $T_{2} \sim\left(l_{p}\right)^{-3}$ while M5-branes have a tension $T_{5} \sim\left(l_{p}\right)^{-6}$.

The simplest way to arrive at a 4-dimensional world is via compactification on a 7-dimensional internal space $\mathcal{M}$. We obtain 2-branes from either the fundamental

M2-branes of $\mathrm{M}$ theory, or by wrapping M5-branes on a 3-cycle $a_{3}$ of the internal space $\mathcal{M}$. Our spectrum of 4 -forms in spacetime comes about in the following way: let $\omega^{i}$ be a basis for $H^{3}(\mathcal{M}, Z)$. We require integer forms so that the resulting 4 -form fields satisfy Dirac quantization. We can then expand $F_{7}$ in this basis,

$$
F_{7}=\widetilde{F}_{i} \wedge \omega^{i}
$$


Note that we get many 4-forms from a generic compactification of this kind. There is a natural geometric picture associated to this expansion. To each form $\omega^{i}$, we can associate a dual 3-cycle on which we wrap an M5-brane to obtain a 2-brane.

After setting the fermions to zero, the 11-dimensional metric satisfies the equation of motion

$$
R_{a b}-\frac{1}{2} g_{a b} R+\frac{1}{6}\left(F_{\text {acde }} F_{b}^{c d e}-\frac{1}{8} g_{a b} F_{c d e f} F^{c d e f}\right)=0 .
$$

The sign of the four-form contribution is fixed by 11-dimensional supergravity. For both kinds of effective 2-brane described above, the contribution of $F$ (or $\widetilde{F}$ ) to the effective cosmological constant is positive. We therefore require a negative bare cosmological constant $\lambda$, as assumed in section 2 .

If $\mathcal{M}$ is circle-fibered then we can reduce $\mathrm{M}$ theory on the fiber to obtain various string compactifications. For example, if we take $\mathcal{M}$ to have the form $S^{1} \times K$ then for a small $S^{1}$ of size $R_{11}$, M theory reduces to type IIA string theory on $K$ [9]. The string scale $\alpha^{\prime}$ and string coupling $g_{s}$ are given in terms of $l_{p}$ and $R_{11}$,

$$
\alpha^{\prime}=l_{p}^{3} / R_{11}, \quad g_{s}=\left(R_{11} / l_{p}\right)^{3 / 2} .
$$

Type IIA string theory contains an NS-NS (Neveu-Schwarz) 3-form field strength $\mathrm{H}_{3}$ which measures fundamental string charge. The magnetic dual $H_{7}=* H_{3}$ measures the charge of NS 5 -branes. These 5 -branes have a tension proportional $1 / g_{s}^{2}$ and so are very heavy at weak string coupling. In addition, there are Ramond-Ramond (RR) field strengths $F_{2}$ and $F_{4}$ (and a non-dynamical $F_{10}$ [17]) together with their 8form and 6-form (and 0-form) magnetic duals. These $(p+2)$-form RR field strengths couple electrically to dynamical Dirichlet $p$-branes [17, 18] through the world-volume action

$$
\int d^{p+1} \xi A_{p+1} .
$$

In type IIA, we therefore have the additional possibility of wrapped D6-branes and D8-branes giving rise to effective 2-branes. In $(9+1)$ dimensions, the low-energy string and $\mathrm{D} p$-brane effective action is

$$
\begin{aligned}
S_{10}= & \frac{1}{2 \kappa_{10}^{2}} \int d^{10} x \sqrt{-G}\left[e^{-2 \phi}\left(R+4(\nabla \phi)^{2}\right)-\sum \frac{1}{2 n !} F_{n}^{2}+\ldots\right] \\
& -T_{p} \int d^{p+1} \xi e^{-\phi} \sqrt{-\operatorname{det} G_{a b}}+\rho_{p} \int d^{p+1} \xi A_{p+1}+\ldots,
\end{aligned}
$$

where $\phi$ is the dilaton, $G_{a b}$ is the induced metric on the brane, and only the relevant bosonic terms are displayed. The tension and charge density of type II Dp-branes is

$$
T_{p}^{2}=\rho_{p}^{2}=\frac{\pi}{\kappa_{10}^{2}}\left(4 \pi^{2} \alpha^{\prime}\right)^{3-p} .
$$

This may be written more conveniently by using the relation $\kappa_{10}^{2}=2^{6} \pi^{7} \alpha^{4}$ and defining the string length $\ell_{s}$ in terms of the fundamental string tension through $T_{\mathrm{F} 1}=$ 
$1 /\left(2 \pi \alpha^{\prime}\right)=2 \pi / \ell_{s}^{2}$. With these conventions,

$$
T_{p}=\rho_{p}=\frac{2 \pi}{\ell_{s}^{p+1}} .
$$

(Note that there is no factor of $1 / g_{s}$ in these expressions because of the conventions employed in Eq. (21).)

The 4-dimensional physical brane tensions and charge densities are proportional to $T_{p}$ and $\rho_{p}$. As we discuss in the next subsections, the exact relations depend on the complicated dynamics of compactification which are not well understood. For this reason, when we consider scenarios in Sec. 旬, we will take a 4-dimensional effective action approach, and simply assume suitable values for the 2-brane tension and charge density. In the rest of this section, however, we explore the possible effects of compactification on these quantities.

There are also other ways of arriving at a 4-dimensional world that do not fit into the above framework. For example, compactifying $\mathrm{F}$ theory [12] on a Calabi-Yau 4-fold [13] naturally gives a large class of $\mathrm{N}=1$ compactifications. The 4 -fold must be elliptically-fibered with a section. If $B$ denotes the 6 -dimensional base of the fibration then over each point of $B$, the structure of the elliptic fibration specifies a torus. By $F$ theory compactified on the 4-fold, we mean type IIB string theory compactified on $B$. However, the complexified string coupling constant $\tau$ is not constant but varies over $B$ in a manner determined by the shape of the torus fiber. Therefore, the string coupling constant is typically not a tunable modulus for these compactifications.

To determine, the spectrum of 2-branes for an F theory compactification, it is natural to use the duality between $\mathrm{M}$ theory on $T^{2}$ and the type IIB string. From this duality, we see that 2-branes arise only from M5-branes. An M5-brane wrapping the elliptic fiber and a 1-cycle in $B$ would look like a D3-brane wrapping the 1-cycle in $B$. There are additional possibilities. For example, an M5-brane with a leg on the elliptic fiber and 3 legs on $B$ can give rise to a 2-brane. From the IIB perspective, it would appear to be a combination of $(p, q)$ 5-branes wrapping a 3-cycle of $B$. In backgrounds such as these with $\mathrm{N}=1$ supersymmetry, we also need to worry about the spacetime superpotential. However, our subsequent discussion will be largely classical. Our aim is to be as simple as possible in order to isolate the aspects of compactifications that are most relevant for the brane nucleation mechanism.

We also note that there is a much broader class of compactifications that involve background fluxes. These compactifications, which are actually generic string compactifications, typically warp spacetime [14, 15, 16]. These backgrounds can potentially give rise to the kind of novel infra-red physics that could make saltatory relaxation a viable mechanism for reducing the cosmological constant.

\subsection{Direct descent}

One might suppose that M2-branes are made to order for saltatory relaxation of the cosmological term. However, as we shall see, they do not have the right properties, 
at least when taken in their straightforward form. The wrapped M5-branes appear more promising.

Let us first consider the case of a D2-brane in 10 dimensions that descends directly to a 2-brane in 4 dimensions. Our discussion will be in the context of 10-dimensional type IIA supergravity compactified on a Calabi-Yau manifold $K$ with string-frame volume $V_{6}$. This compactification preserves $\mathrm{N}=2$ supersymmetry. The physical effective tensions and charge densities are then determined in 4-dimensional Einstein frame, where the gravitational action takes the conventional Einstein-Hilbert form. This frame follows after performing the Weyl rescaling $g_{\mu \nu} \rightarrow e^{2 \phi} g_{\mu \nu}$ on the 4-dimensional metric $g_{\mu \nu}$. The 4-dimensional action is then

$$
\begin{aligned}
S_{4}= & \frac{V_{6}}{2 \kappa_{10}^{2}} \int d^{4} x \sqrt{-g}\left[R-2(\nabla \phi)^{2}-\sum \frac{1}{2 n !} e^{-2(n-2) \phi} F_{n}^{2}+\ldots\right] \\
& -T_{p} \int d^{p+1} \xi e^{p \phi} \sqrt{-\operatorname{det} g_{a b}}+\rho_{p} \int d^{p+1} \xi A_{p+1}+\ldots .
\end{aligned}
$$

The 4-dimensional effective tension is $\left.\tau_{p}\right|_{4 \mathrm{D} \text {,eff }}=T_{p} g_{s}^{p}$, where $g_{s}=e^{\langle\phi\rangle}$ is the string coupling. To determine the effective charge density, note that the $F_{n}^{2}$ kinetic terms are not canonically normalized. Upon restoring the normalization, we find a 4-dimensional effective charge density of $\left.\rho_{p}\right|_{4 \mathrm{D} \text {,eff }}=\sqrt{2} \rho_{p} g_{s}^{p} / M$, where the reduced Planck mass is fixed by $M^{2}=V_{6} / \kappa_{10}^{2}$. The tension to charge density ratio, in Planck units, is then $x=1 / \sqrt{2}$, as we expect for BPS branes.

For the 2-brane case of interest,

$$
\left.\rho_{2}\right|_{4 \mathrm{D}, \mathrm{eff}}=\frac{2 \sqrt{2} \pi g_{s}^{2}}{M \ell_{s}^{3}} .
$$

Clearly, to obtain a sufficiently small charge density, we must have extreme values for $\ell_{s}$ and/or $g_{s}$. For example, for the canonical choice of string scale $\ell_{s} \simeq\left(10^{17} \mathrm{GeV}\right)^{-1}$, we find that a charge density of $\rho_{2} \lesssim 10^{-90}$ requires $g_{s} \lesssim 10^{-44}$. Alternatively we could take $\ell_{s}$ to be a larger length scale. These non-canonical cases include the 'large extra dimension' scenario [25] with $g_{s} \sim 1$ and some number of sub-millimeter dimensions. However, given the success of quantum field theory at colliders such as LEP and the Tevatron, $\ell_{s} \lesssim(\mathrm{TeV})^{-1}$ at the very best. Although an improvement, this still requires a tiny string coupling $g_{s} \lesssim 10^{-23}$ to generate a sufficiently small $\rho_{2}$. It is possible that this string coupling is unrelated to the gauge couplings of the Standard Model; for example, the Standard Model may arise from some non-perturbative sector of string theory. However, if $g_{s}$ is related to the Standard Model gauge couplings in a straightforward way, it is difficult to understand how to accommodate the Standard Model in such an extreme corner of string theory moduli space, much less gauge coupling unification with $\alpha_{\text {unif }} \simeq 1 / 25$ [26]. 


\subsection{Degenerating cycles}

A more promising alternative to direct descent branes are branes wrapped on homology cycles. These branes become tensionless when the volume of these cycles approaches zero classically, as for conifolds [27]. For compactifications with $N \geq 2$ supersymmetry, these branes give rise to BPS states and so quantum corrections do not change this conclusion.

Specifically, if a $p$-brane of tension $T_{p}$ wraps a $k$-cycle $a_{k}$ of the compactification manifold, where $k \leq p$ and the volume of $a_{k}$ is $\operatorname{Vol}\left(a_{k}\right)$, then the result in the effective 4 -dimensional theory is a $(p-k)$-brane of tension $\tau_{(p-k)} \sim T_{p} \cdot \operatorname{Vol}\left(a_{k}\right)$. In particular, for an effective 2-brane in 4 dimensions coming from the wrapping of a $\mathrm{D} p$-brane on a cycle $a_{p-2}$, the Einstein frame effective tension is

$$
\left.\tau_{2}\right|_{4 \mathrm{D}, \mathrm{eff}}=T_{p} \operatorname{Vol}\left(a_{p-2}\right) g_{s}^{2} .
$$

If $\operatorname{Vol}\left(a_{p-2}\right)$ approaches zero, i.e., $a_{p-2}$ is a degenerating cycle, a nearly tensionless object exists in the 4-dimensional theory. The analogous formula for the tension of a wrapped NS 5-brane just differs by a factor of $g_{s}$. This is consistent with the higher-dimensional quantization rules for brane properties.

What determines the charge density? As we saw in the case of the direct reduction of an M2-brane to a D2-brane, the kinetic terms for the 3-form gauge-field become moduli-dependent. The term,

$$
\int d^{p+1} \xi A_{p+1}
$$

is reduced on integer classes and so contains no moduli dependence. For purposes of determining the scaling of the charge density with the moduli, we only need the behavior of the moduli space metric near the singularity. At the level of classical geometry, this is determined by the behavior of the Weil-Petersson metric. For $\mathrm{N}=2$ compactifications, this metric behavior is not changed by quantum corrections, although it is certainly renormalized in situations with less supersymmetry. Let us take the case of the conifold where an $S^{3}$ shrinks to zero size in $K$. We choose coordinates for the moduli space so that the singularity is located at $Z=0$. The tension of a wrapped brane goes like,

$$
\left.\tau_{2}\right|_{4 \mathrm{D}, \mathrm{eff}} \sim|Z| .
$$

However, the moduli space metric scales like $\ln (Z)$ and so the charge density behaves like [28],

$$
\left.\rho_{2}\right|_{4 \mathrm{D}, \mathrm{eff}} \sim 1 / \sqrt{|\ln (Z)|} .
$$

Thus although the tensions can be vanishingly small, the charge densities generated are only slightly smaller than in the direct descent case. We would therefore need to stabilize $Z$ at extremely small values in order to obtain an acceptably small charge density.[]

\footnotetext{
${ }^{2}$ We thank J. Polchinski for correcting an error in an earlier version of this paper, and J. Maldacena for discussions.
} 
The log-singularity in the metric has a simple space-time interpretation as arising from integrating out a massless hypermultiplet [27]. We desire a singularity at finite distance in the moduli space whose behavior is worse than that of a conifold. It is not hard to see that in the context of $\mathrm{N}=2$ compactifications, such singularities cannot arise by simply tuning vector multiplet moduli. For example, one can try generalizations of the conifold with multiple simple nodes. These singularities are all at finite distance in the moduli space. However, in each case and in general, it appears that gravity can be decoupled. The resulting theory of vector multiplets has a moduli space metric with singularities than metrically cannot be worse than logarithmic by standard non-renormalization theorems.

Too little is currently known about the hypermultiplet moduli space to decide whether a singularity with the right metric behavior exists. Some results on classifying singularities at finite distance have appeared in [29]. However, when we relax the condition of $\mathrm{N}=2$ supersymmetry and consider the far larger class of $\mathrm{N}=1$ compactifications, it seems far more likely that a sufficiently bad singularity exists (classically). This is quite exciting since it connects the possibility of novel infra-red physics with a mechanism for reducing the cosmological constant. A recent $\mathrm{F}$ theory example with unusual infra-red physics arising from a bad singularity appeared in [30]. This kind of example certainly has the right qualitative features, and it actually seems quite hard to rule out the existence of a compactification with the features we desire. Clearly more work along these lines is needed.

We have yet to discuss supersymmetry breaking. One natural mechanism worth mentioning in this context breaks supersymmetry by turning on $R R$ and $N S$ fluxes on $K$. For example, we can give $(3+1)$-Lorentz invariant expectation values to the RR fields $F_{n}$ by taking,

$$
\left\langle F_{2}\right\rangle=v_{2}^{i} \alpha_{i}^{(2)}, \quad\left\langle F_{4}\right\rangle=v_{4}^{i} \alpha_{i}^{(4)}+v_{4} \varepsilon^{(4)} .
$$

The $\alpha_{i}$ are harmonic forms on $K$, and $\varepsilon^{(4)}$ is the spacetime volume element. If we take the 10-dimensional Poincaré duals of these expectation values $v_{(4,2)}^{i}$, we then find expectation values of the general form $F=v_{(4,2)}^{i} \varepsilon^{(4)} \wedge \widetilde{\omega}^{(2,4)}$, which couple magnetically to the $\mathrm{D} p$-branes. Let us denote the expectation values collectively by $v_{a}$.

Generically, when $v_{a} \neq 0$, supersymmetry is broken [19, 20, 21, 22, 23], and a scalar potential is generated that depends on the Calabi-Yau moduli $t_{\alpha}$ and the expectation values $v_{a}$. What is interesting to us, however, is that the potential naturally tends to drive us to singular compactifications. In some examples involving just RR fluxes, two types of critical point for the scalar potential have been found [20, 21]: either the Calabi-Yau runs away to infinite volume, or the theory is driven to conifold-like points where homology cycles of $K$ degenerate (classically approach zero volume). The minima tend to either break supersymmetry completely or restore the full $\mathrm{N}=2$. Moreover, since the configurations with $v_{a} \neq 0$ are not iso-potential, there are dynamical processes whereby the values of the $v_{a}$ 's change. The $v_{a}$ 'discharge' by the nucleation and expansion of charged membranes, the D-branes of string theory. 
Classically, the vacuum expectation values $v_{a}$ of the $\mathrm{RR}$ fields can vary continuously. Quantum mechanically, they satisfy quantization rules that follow from the quantization of brane properties, which are standard results in string theory [24. These brane properties are fixed, given information about the compactification and the parameters of our low-energy effective Lagrangian.

There is an additional issue concerning this means of supersymmetry breaking that deserves mention. In the context of $\mathrm{M}$ theory on $K \times S^{1}$, a class of 2-branes arise from M5-branes wrapping 3-cycles. In type IIA, these 2-branes arise from either D4-branes wrapping 2-cycles of the compactification space $K$, or from NS 5-branes wrapping 3cycles of $K$. The two cases are quite different. In the case of the wrapped D4-branes, we must also consider D2-branes which can also wrap the shrinking 2-cycle. These wrapped branes give rise to particle states with mass $\tau_{0} \sim \operatorname{Vol}\left(a_{2}\right) / \ell_{s}^{3} \rightarrow 0$. With multiple collapsed nodes, we will find an interacting gauge theory in $3+1$-dimensions. In this case, it seems possible that the wrapped D4-branes will correspond to collective excitations of the gauge theory. This is not an issue, however, for the wrapped NS 5-branes since there are no BPS D3-branes in type IIA string theory. These wrapped NS-branes give rise to inherently non-perturbative stringy excitations.

The phenomenon of classically vanishing tensions arising from branes wrapped on degenerating cycles is intriguing. It is also worth noting that the classical phenomenon of true degeneration and corresponding tensionless, or massless, states is typically not realized in the full quantum theory. Instead the effective $(3+1)$-dimensional 2-brane will have a dynamically generated non-perturbative tension, which may be exponentially small. It is known that in some cases a tension is generated from the dynamics of the would-be massless particle states arising from a D2-brane wrapping the 2-cycle. These particle states realize a non-Abelian gauge theory, presumably in a sector hidden with respect to the Standard Model, whose low-energy non-perturbative dynamics can break supersymmetry. (This sector is conceptually distinct from hidden sectors postulated to provide supersymmetry breaking for the supersymmetric Standard Model.) This leads to a potential for the volume modulus of the cycle, which stabilizes it at a scale $\Lambda \sim \exp \left(-8 \pi^{2} / b_{1} g_{Y M}^{2}\right) M$ [31, 23, 22, 32]. Once the cycle is stabilized at this small scale, membranes wrapping this cycle have a tension that is also proportional to $\Lambda$ and thus can be very small, even for $g_{Y M} \sim 1$. The precise conditions under which very small tensions occur deserve much further study.

Some years ago, one of us made the numerical joke 33]

$$
\Lambda_{\text {obs }} M^{2} \sim\left(e^{-\pi / \alpha_{\text {unif }}} M\right)^{4},
$$

with $\alpha_{\text {unif }} \simeq 1 / 25$. Our present considerations suggest an intimate connection between non-perturbative effects and the value of the observed cosmological term, which could conceivably lead to a relation of just this form.

\section{Saltation in String Theory: Density of States}




\subsection{Multi-bounce properties}

An essentially new feature is introduced by multi-bounce solutions arising from coincident branes. Such coincident branes support low-energy internal degrees of freedom. Thus there are potentially large density of states factors accompanying their nucleation. Calculations [34, 35] performed in the context of checking duality between type I and heterotic $\mathrm{SO}(32)$ string theory demonstrate that D-branes do make contributions that can be interpreted semi-classically as incorporating degeneracy factors reflecting the non-Abelian structure of coincident D-branes. Another aspect of this is that many coincident branes with large total charge can be described in appropriate limits as 'black' objects, similar to black holes, with event horizons, and with associated Bekenstein-Hawking (BH) entropy [36].

Consider first the case of $k$ coincident D3-branes. Such a configuration possesses a $\mathrm{U}(k)$ super Yang-Mills (SYM) gauge theory on its world-volume. In the limit where the interactions with the bulk string theory are weak, and where the temperature (or excitation energy) of the SYM is small, one can compute the entropy of this system.

When the effective SYM gauge coupling $g_{\mathrm{eff}}^{2} \simeq k g_{\mathrm{YM}}^{2}$ is small, the entropy of the gas of massless gauge bosons and their superpartners at temperature $T$ is simply

$$
S_{3}=\frac{2 \pi^{2}}{3} k^{2} V T^{3}
$$

where $V$ is the spatial volume of the 3-branes. What happens when the effective coupling is large? In this case one can use the type IIB supergravity solution describing the $k$ coincident 3-branes. These classical solutions with the asymptotic geometry and quantum numbers appropriate for $k$ coincident 3-branes contain a non-extremality parameter upon which their masses and horizon areas depend. If we associate the area of the horizon with BH entropy, we can derive a temperature by taking an appropriate derivative. By this procedure, the supergravity picture yields the strong coupling form of the entropy. In this case, the entropy agrees with the preceding weak coupling formula up to a numerical prefactor $3 / 4$, which is then a prediction for the strong coupling behavior of the theory.

For $k$ coincident D2-branes the UV theory (in the decoupling limit) is again a weakly-coupled $\mathrm{U}(k)$ SYM theory, so the UV entropy again scales as $k^{2}$. However we are interested in the IR entropy since, as we will argue in the next subsection, the physically motivated temperatures are the ambient de Sitter temperatures which are small (vanishingly small as $\Lambda_{O} \rightarrow \Lambda_{\text {obs }}$ ). In the IR the SYM theory on the $(2+1)$ dimensional world-volume becomes strongly coupled, so one must switch over to the supergravity description. As shown in Ref. [37], the theory flows in the far IR to that of the M2 brane with BH entropy inferred from the horizon area given by [36]

$$
S_{2} \simeq k^{3 / 2} A T^{2}
$$

with $A$ the 2-brane area. This strongly suggests that such strongly-coupled brane configurations support $\mathcal{O}\left(k^{3 / 2}\right)$ light degrees of freedom, though the physical nature 
of these degrees of freedom remains somewhat mysterious. Thus the probability for a semi-classical process involving $k$ coincident D2-branes is multiplied by a density of states factor of the form $N_{k} \sim \exp \left(k^{3 / 2} A T^{2}\right)$ in the IR limit $T \rightarrow 0$.

The branes of interest to us are effective 2-branes arising from the wrapping of, say, a D4-brane on a 2-cycle, $a_{2}$, or in the M theory picture an M5-brane wrapped on $S^{1} \times a_{2}$. Forgetting for a moment about the wrapping on a cycle, $k$ coincident D4-branes have a (4+1)-dimensional SYM theory which now flows to a free theory in the IR, so the entropy would scale as $k^{2} V_{4} T^{4}$ (the M5-branes have a rather unusual, and microscopically not fully understood, scaling $S \sim k^{3}$ ). In the case of the wrapped branes, the excitations in the wrapped directions are massive and not excited at the low temperatures we consider, so the entropy scales as $S \sim A T^{2}$ with $A$ being the area of the 2-brane in our extended (3+1)-dimensional spacetime. Such behavior is correct until the temperature $T$ falls below the inverse linear size $1 / b$ of the nucleated brane, below which point the density of states factor just counts the number of zero modes. From the preceding discussion, we expect the scaling of the entropy with $k$ in this case to lie between $k^{2}$ and $k^{3 / 2}$. A more exact treatment requires an additional analysis of the way in which the exponent scales as we approach the IR, as the physically motivated temperature is small but non-zero, and it is incorrect to scale infinitely far into the IR. In the case of a degenerating cycle, however, since the near degeneration implies that the world-volume theory of the effective 2-brane in $(3+1)$ dimensions has a bare coupling that is large, we regard the lower value $\beta=3 / 2$ as being more likely.

In any case, if we accept this reasoning, the probability for a semi-classical process involving $k$ coincident 2 -branes must be multiplied by an appropriate density of states factor of the form

$$
N_{k} \sim \exp \left(k^{\beta} A T^{2}\right),
$$

for an appropriate temperature $T$, with an exponent $\beta$ that likely lies between $\beta=2$ and $\beta=3 / 2$. (Although we will not utilize it here, there might also be the possibility of $k^{3}$ scaling in the M5-brane limit.) As we will show below, a larger $\beta$ exponent implies more complete saltation, so we will adopt the more 'conservative' value of $\beta=3 / 2$ as our canonical choice.

\subsection{Temperature: ambiguity, black hole analogy}

The only temperatures intrinsic to our scenario are the de Sitter temperatures

$$
T_{O, I}=\frac{H_{O, I}}{2 \pi}=\frac{1}{2 \pi} \sqrt{\frac{\Lambda_{O, I}}{3}} .
$$

Ambient ordinary matter might supply a much higher temperature (see Sec. 6), but the branes are in very poor thermal contact with it. (Ambient D-matter, i.e., the light particles mentioned above, might supply a better coupled temperature bath, but we shall not pursue this possibility.) 
If the initial cosmological term is much larger than the change brought about by the $k$-bounce,

$$
\Lambda_{O} \gg k \rho_{2} c_{O},
$$

then the de Sitter temperatures before and after nucleation are almost identical, $T_{O} \simeq T_{I}$, and we may use either one in calculating the density of states factor.

In the case that a given transition produces large changes in the effective cosmological constant, an ambiguity arises. One possibility is that the temperature scale for tunneling from a highly curved (high temperature) de Sitter space to a less curved de Sitter space (or even to a flat or anti-de Sitter space) is substantially set by the high temperature. In this case one would take

$$
T \sim T_{O}
$$

in the density of states factor of Eq. (30). We will consider the dynamics of this possibility in Sec. 5.1.

However, when the change in the nominal de Sitter temperature is comparable to the temperature itself, the thermal description of the tunneling process is internally inconsistent. A similar situation has been encountered before, in black hole physics [38]. The problem arises in its most acute form for near-extremal holes, as the temperature approaches zero. If one uses the temperature of the initial hole, one finds a significant probability for radiating a quantum that will take the hole past extremality to a naked singularity. A more refined analysis [39, 40] shows that radiation is not thermal with regard to the initial temperature, and in particular that radiation beyond extremality is forbidden.

If we make an analogy between maximally homogeneous cosmologies and black holes based on their temperatures, then de Sitter spaces correspond to ordinary holes, flat space corresponds to an extremal hole, and anti-de Sitter spaces to naked singularities. This analogy suggests, in view of the previous paragraph, that we should not consider finite temperature branes that mediate transitions from de Sitter to antide Sitter spaces. A crude working hypothesis, which interpolates smoothly to this suggestion, is that in the density of states factor of Eq. (30), we should, instead of $T \sim T_{O}$, employ the geometric mean of the de Sitter temperatures

$$
T \sim \sqrt{T_{O} T_{I}} .
$$

The dynamics of this possibility is explored in Sec. 5.2.

\subsection{A catastrophe for $T \sim T_{O}$ ?}

If the analogy to black hole results holds, tunneling to (and through) anti-de Sitter space is excluded. On the other hand, if the temperature for transitions is effectively set by $T_{O}$, multi-bounce transitions with arbitrarily large $k$ are possible and must be considered. 
The action for $k$-bounce tunneling is given by Eqs. (7)-(11), with $\rho_{2} \rightarrow k \rho_{2}$ and $\tau_{2} \rightarrow k \tau_{2}$. The action is monotonically increasing as $k$ increases, and has the limiting behavior

$$
B=\frac{24 \pi^{2} M^{2}}{\Lambda_{O}}, \quad k \rightarrow \infty .
$$

The limit $k \rightarrow \infty$ therefore appears problematic, since $P \sim N_{k} e^{-B}$ approaches a constant for large $k$. Note, however, that as $k \rightarrow \infty$, the bubble size $b \sim \frac{1}{k} \rightarrow 0$. Thus, the apparent 'instability' is toward creation of highly curved branes. We do not expect the action of Eq. (回) to be valid in the regime where the brane curvature far exceeds the brane tension. P Presumably, a calculation of the tunneling probability with the degrees of freedom appropriate for this regime will be well-behaved.

\section{Scenarios}

Let us now gather the pieces and attempt to envisage how - and whether! - they may be assembled into a complete scenario.

The cosmological constant evolves from some initial value through multi-bounce transitions. The probability for such transitions is

$$
P \sim e^{D} e^{-B} .
$$

The bounce action $B$ is given by Eqs. (7)-(11), with $\rho_{2} \rightarrow k \rho_{2}$ and $\tau_{2} \rightarrow k \tau_{2}$, where $k$ is the bounce number. The density of states prefactor is specified by $D=k^{\beta} A T^{2}$, where $A=4 \pi b^{2}$ is the 2-brane area, and $T$ is the temperature. For concreteness, we will assume the low 'conservative' value of $\beta=3 / 2$, but the qualitative features of the following analysis hold more generally, for example, for $\beta=2$ or larger.

As noted above, the temperature $T$ is not under good theoretical control. We will therefore explore both of the broad alternatives mentioned previously.

\section{$5.1 T \sim T_{O}$, 1-step relaxation}

We first consider the possibility that the temperature is given by the scale of the initial (outside) de Sitter temperature, so $T \sim T_{O} \sim \sqrt{\Lambda_{O}}$. The tunneling probability from a given background configuration is then fixed in terms of the initial effective cosmological constant $\Lambda_{O}$, the initial field strength $c_{O}$, and the 2-brane charge density and tension, parameterized by $\rho_{2}$ and $x \equiv \tau_{2} / \rho_{2}$.

We begin with some bare cosmological constant $\lambda<0$. Assume that the initial field strength gives a similar contribution to the effective cosmological constant, so $\Lambda_{O}, c_{O}^{2} \sim|\lambda|$. We assume also a very small charge density $\rho_{2} \sim \Lambda_{\text {obs }} / \sqrt{|\lambda|}$, consistent with the naturalness condition discussed in Sec. 2.2, and $x \sim 1$.

\footnotetext{
${ }^{3}$ We thank R. Sundrum for emphasizing this point to us.
} 
With such initial conditions and brane properties, the maximal bounce action is $B \sim 1 /|\lambda|$, while the degeneracy factor may be as large as $D \sim \lambda^{2} / \Lambda_{\text {obs }}^{2}$. Recall that $\Lambda_{\text {obs }} \sim 10^{-120}$, while $\lambda$ is plausibly in the range of 1 (for Planck scale cosmological constants) to $10^{-60}$ (for low-scale supersymmetry breaking at the $\mathrm{TeV}$ scale). Thus, the degeneracy enhancement overpowers the bounce action suppression, and tunneling proceeds rapidly.

It is not difficult to show that $D$ is maximized for $k \rho_{2} \sim c_{O}$, i.e., for field strength step sizes of the right order to neutralize the field strength contribution to the effective cosmological constant. Indeed the most probable tunneling events nucleate bubbles of deep anti-de Sitter space. Such events produce small, short-lived interior universes, so the meaning of 'probable' in this context must be carefully qualified. Among universes that live a long time and even remotely resemble ours, the exponentially most favored are those closest to having zero effective cosmological term.

This scenario invokes a form of the anthropic principle. It is a uniquely weak one, however, in the following sense. Anthropic bounds on the cosmological term are highly asymmetrical [1]. For positive cosmological terms, the formation of sufficiently large gravitational condensations requires cosmological terms below $\sim 100$ in units of $\rho_{c}$, the critical density. For negative cosmological terms, the lifetime of the universe requires cosmological terms roughly above -1 . Thus if the spacing between allowed near-zero saltatory values of the cosmological term in units of $\rho_{c}$ is, say, 3 and allows the values $\ldots,-2,1,4, \ldots$, then among values that can be experienced by sentient observers, 1 is by far the most likely.

Now suppose such a transition to nearly flat space has occurred. As discussed in Sec. 2.3, absolute stability is possible only for anti-de Sitter spaces, and only for extremely large $x$. However, absolute stability is not required on empirical grounds. We need only require that the effective value of the cosmological term is at present stable on cosmological time scales. For a starting effective cosmological constant of $\Lambda_{O} \sim \Lambda_{\text {obs }}$, the degeneracy factor is highly suppressed by small $T$, and the bounce action is dominant. The stability of the vacuum is then determined solely by $B$.

Since the bounce action can become truly infinite for slightly anti-de Sitter spaces, we anticipate, by continuity, that it can become very large even for slightly de Sitter spaces. The least suppressed transition (and so most dangerous from the point of view of vacuum instability) is that mediated by $k=1$. For small $\rho_{2}$ and small $\Lambda_{O}$, the $k=1$ bounce action is

$$
B \approx \frac{27 \pi^{2} x^{4}}{2}\left[\frac{\rho_{2}}{c_{O}^{3}}-\frac{\rho_{2}^{2}}{c_{O}^{2} \Lambda_{O}}\right], \quad \rho_{2}, \sqrt{\Lambda_{O}} \ll c_{O} .
$$

Neglecting numerical factors, we find

$$
B \sim x^{4} \Lambda_{\mathrm{obs}} / \lambda^{2}
$$

If we now take $|\lambda| \sim 1$, then the action is very small, and there is no effective stability. 
On the other hand, if supersymmetry is broken at a low scale, then we expect $|\lambda| \ll 1$. Let us inquire when $B \gtrsim 1$. This translates into

$$
\left|\lambda_{\text {halting }}\right| \lesssim x^{2} \sqrt{\Lambda_{\text {obs }}}
$$

or, restoring the mass units,

$$
\left|\lambda_{\text {halting }}\right| M^{2} \lesssim x^{2}\left(2 \times 10^{-3} \mathrm{eV}\right)^{2}\left(2.4 \times 10^{18} \mathrm{GeV}\right)^{2} \sim x^{2}(2 \mathrm{TeV})^{4} .
$$

In models where supersymmetry breaking is transmitted with little suppression to the Standard Model fields (or is even present in the observable sector itself), it is reasonable to expect the supersymmetry breaking scale to set the scale of the bare cosmological constant, so

$$
M_{\text {weak }}^{4} \sim M_{\mathrm{SUSY}}^{4} \sim\left|\lambda_{\text {halting }}\right| M^{2} .
$$

Given the present experimental lower bounds on the supersymmetry breaking scale, we find then that the stability of the vacuum in this scenario requires low scale supersymmetry breaking, and relates the cosmological constant, Planck, and weak scales according to

$$
M_{\text {weak }}^{2} \sim\left(10^{-3} \mathrm{eV}\right)\left(M_{\text {Planck }}\right),
$$

in accord with observation.

In a more careful analysis, one may require $B \gg 1$ for stability. However, the required supersymmetry breaking scale will not differ significantly from the above estimates, as $B$ goes as the inverse 8th power of the energy scale appearing in $|\lambda| M^{2}$. Finally, note that we have assumed the qualitative validity of the bounce action Eq. (7)-(11) throughout this section. For large $\lambda \gg 10^{-60}$, this may not be appropriate, as the brane curvature may be much greater than the brane tension. However, we have checked that in the case of greatest interest to us with $\lambda \sim 10^{-60}$, the brane curvature never greatly exceeds the brane tension, and the analysis above is under reasonable control.

\section{$5.2 T \sim\left(T_{I} T_{O}\right)^{1 / 2}$, multi-step relaxation}

Motivated by the black hole analogy, we now consider an effective temperature that is the geometric mean of the initial and final de Sitter temperatures. In this case, tunneling to (and through) anti-de Sitter space is forbidden by fiat. However, the requirements of rapid tunneling to the observed cosmological constant and its stability are non-trivial constraints, and we now investigate their implications.

As in the previous scenario, we consider initial conditions $c_{O}^{2}, \Lambda_{O} \sim|\lambda|$. Now, however, the density of states factor $D$ is typically maximized for $\Lambda_{I}$ within an order of magnitude of $\Lambda_{O}$. To see this, a very rough estimate may be obtained by neglecting the bubble radius dependence on $k$ and approximating $\Lambda_{O}-\Lambda_{I}=\left(2 \rho_{2} c_{O}-\rho_{2}^{2}\right) / 2 \sim \rho_{2} c_{O}$. We then have $D \propto \sqrt{\Lambda_{O} \Lambda_{I}}\left(\Lambda_{O}-\Lambda_{I}\right)^{3 / 2}$, which is maximized for $\Lambda_{I}=\frac{1}{4} \Lambda_{O}$. 
For $\Lambda_{I} \sim \Lambda_{O}$,

$$
D_{\max } \sim \frac{\Lambda_{O}^{5 / 2}}{|\lambda|^{7 / 4} \rho_{2}^{3 / 2}} .
$$

It is not hard to verify that this degeneracy factor dominates the bounce suppression when $\Lambda_{O} \sim|\lambda|$. Thus, initially the effective cosmological constant tunnels rapidly as in the previous scenario, but in contrast to the previous case, the cosmological constant relaxes through several steps, with values roughly following a geometric series.

The effective cosmological constant will relax as described until $\Lambda_{O} \ll c_{O}^{2}$, when Eq. (37) holds. At this point, the condition that tunneling continue is the requirement $D_{\max } \gtrsim B$, or, since $B \sim \Lambda_{O} / \lambda^{2}$,

$$
\Lambda_{O}^{3 / 2} \gtrsim|\lambda|^{-1 / 4} \rho_{2}^{3 / 2} .
$$

For vanishing $\rho_{2}$, tunneling may continue to arbitrarily small $\Lambda_{O}$. However, if we require stability from $B \gtrsim 1$, we find, from Eq. (37),

$$
\rho_{2} \gtrsim c_{O}^{3} \sim|\lambda|^{3 / 2},
$$

so $\rho_{2}$ cannot be arbitrarily small. Combining Eqs. (44) and (45), we find that tunneling stops when

$$
\Lambda_{O} \gtrsim|\lambda|^{4 / 3} .
$$

Thus, even for $|\lambda| \sim 10^{-60}$, although the effective cosmological constant is reduced by a factor of $10^{20}$, one membrane cannot suppress it to the observed value.

In general, however, it is important to note that several different 2-branes with various fundamental charge densities may be expected to arise. Suppose that another brane begins nucleating as the first membrane reaches its endpoint. The initial conditions for this new membrane are identical to those for the first brane, except that now the role of the initial bare cosmological constant is played by $\Lambda_{O} \sim|\lambda|^{4 / 3}$. For appropriate charge densities, $n$ branes may reduce the cosmological constant to $|\lambda|^{(4 / 3)^{n}}$. For $|\lambda| \sim 10^{-60}$, three branes are sufficient to reduce the cosmological constant to its observed value.

So far we have considered only the 'conservative' $\beta=3 / 2$ case. For larger values of $\beta$ more complete relaxations of the cosmological term are possible. For general $\beta$, a single membrane may relax the cosmological constant to $\Lambda_{O} \gtrsim|\lambda|^{2-\beta^{-1}}$. Thus, even for the $\beta=2$ case, only two stages are required. Note also that in these multibrane scenarios, in principle quite complex dynamics can arise, with periods of slow relaxation interspersed with more rapid changes.

\section{Summary and Discussion}

On very general grounds, it is appealing to think that relaxation of the cosmological term might be associated with very special degrees of freedom that have no conven- 
tional couplings to matter, and no conventional kinetic energy, but respond only to $3+1$ dimensionally uniform form fields and, of course, to gravity. Several difficulties in such an approach must be addressed. The phenomenologically required energy scale is very small and not easily manufactured out of conventional energy scales. In addition, in any reasonable scenario, the cosmological constant must relax sufficiently quickly from high scales, but must be stable on cosmological time scales at its present value.

String theory provides a promising microscopic framework for such a mechanism. The necessary degrees of freedom are naturally supplied by string/M theory branes, and the dependence of brane properties on compactification appears, in principle, to be capable of producing a very small scale. We have also identified a candidate mechanism, the enhancement of multi-step jumps due to large density of states factors, which typically leads to large tunneling probabilities. Finally, as we have seen, the absolute stability of certain anti-de Sitter universes implies that near flat universes may be also be sufficiently stable.

We have considered two representative scenarios differing in the treatment of the effective temperature entering the density of states factor. In the simplest scenario, with $T \sim T_{O}$, the exponentially most probable transition, excluding extremely shortlived universes, is to universes that are most nearly flat. By requiring that this new vacuum be sufficiently stable, we derived a non-trivial constraint for a mechanism of this kind. This constraint provides an intriguing relationship, between the supersymmetry breaking scale and the geometric mean of the present-day effective cosmological constant and Planck scales:

$$
M_{\text {SUSY }}^{2} \lesssim\left(10^{-3} \mathrm{eV}\right)\left(M_{\text {Planck }}\right)
$$

Large supersymmetry breaking scales are thereby excluded, and the largest possible scale is plausibly though not necessarily satisfied in Nature.

In reaching this relation, we have assumed the bare cosmological constant to be of order the supersymmetry breaking scale. Indeed, while the bare cosmological term appears as a free parameter in supergravity, in string/M theory, phenomenologically interesting models with unbroken supersymmetry have zero cosmological term. In this context, it is therefore reasonable to expect that the relevant scale for the bare cosmological term is indeed the supersymmetry breaking scale. Many models of supersymmetry breaking invoke 'hidden sectors' with a characteristic mass scale much larger than the $\mathrm{TeV}$ scale. Unless the hidden sector contribution to the bare cosmological term is somehow suppressed to this $\mathrm{TeV}$ scale, in our simplest scenario the vacuum will be unstable.

Alternatively, an analogy with black holes suggests a richer dynamics, in which flat space plays a distinguished role and tunneling to anti-de Sitter space is forbidden. Within this circle of ideas, and in contrast to the previous scenario, we found that the cosmological constant relaxes through a several jumps, roughly following a geometric series. The constraint of stability limits the range over which the cosmological 
constant may be relaxed by any given membrane. However, two or more types of branes with radically different scales may relax the cosmological constant to within observational bounds, and appeal to the anthropic principle may be avoided.

Our work so far is very seriously incomplete, in that we have not attempted to incorporate it into a realistic (e.g., Friedmann-Robertson-Walker) cosmological model including matter. Thus, in particular, we have not addressed the dynamics of relaxation following a phase transition. There is a potential problem here, since, if after relaxation to zero effective cosmological term a later matter phase transition drives it negative, recovery may be difficult. We note also that the existence of very light membrane degrees of freedom may have a variety of observational and experimental consequences. We reserve discussion of these issues for a future publication.

We also require, for our dynamics, compactification schemes that produce the desired brane properties. Most model building in string/M theory has been based, implicitly or explicitly, on the paradigm of minimizing a potential. This has always been problematic within a quantum theory of gravity, but it was not clear what could replace it. The saltatory mechanism suggests a different principle, based on the dynamics of relaxation of the cosmological term. Whether this principle, or any other, is powerful enough to select uniquely a vacuum as complex as the one we observe remains to be seen.

\section{Acknowledgments}

We are very grateful to Luis Alvarez-Gaume, Paul Aspinwall, Raphael Bousso, Amit Giveon, Alex Kusenko, Juan Maldacena, Emil Martinec, Peter Mayr, Greg Moore, Yaron Oz, Joe Polchinski, Raman Sundrum, and Claudio Teitelboim for discussions. The work of JLF is supported in part by the Department of Energy under contract DE-FG02-90ER40542 and through the generosity of Frank and Peggy Taplin. JMR wishes to thank the Alfred P. Sloan Foundation for the award of a Fellowship, and the US Department of Energy for an Outstanding Junior Investigator Award. The work of SS is supported in part by the William Keck Foundation and by NSF grant No. PHY-9513835. The work of FW is supported in part by the Department of Energy under contract DE-FG02-90ER40542 and by the National Science Foundation under grant PHY-9513835.

\section{Note}

As this manuscript was being completed, we learned of independent work by Bousso and Polchinski [41] proposing quite a different scenario for fixing the cosmological term in string theory through a generalization of the Brown-Teitelboim mechanism. They do not utilize degenerating cycles nor enhanced density of states factors, and instead invoke the anthropic principle in an essential way. We thank them for conversations 
regarding our respective approaches.

\section{References}

[1] S. Weinberg, Rev. Mod. Phys. 611 (1989).

[2] S. Coleman, Phys. Rev. D15 2929 (1977), Erratum, Phys. Rev. D16 1248 (1977);

C. Callan and S. Coleman, Phys. Rev. D16 1762 (1977).

[3] S. Coleman and F. de Luccia, Phys. Rev. D21 3305 (1980).

[4] L. Abbott, Phys. Lett. B150 427 (1985).

[5] J. Brown and C. Teitelboim, Phys. Lett. B195 177 (1987);

Nucl. Phys. B297 787 (1988).

[6] R. Bousso and A. Chamblin, Phys. Rev. D59 063504 (1999), hep-th/9805167;

M. S. Bremer et al., Nucl. Phys. B543 321 (1999), hep-th/9807051.

[7] See, e.g., S. Hawking and N. Turok, Phys. Lett. B432 271 (1998), hep-th/9803156.

[8] J. D. Cohn, astro-ph/9807128; S. Carroll, astro-ph/0004075.

[9] P. K. Townsend, Phys. Lett. B350 184 (1995), hep-th/9501068;

E. Witten, Nucl. Phys. B443 85 (1995), hep-th/9503124.

[10] For excellent introductory lectures see:

P. K. Townsend, hep-th/9612121;

M. J. Duff, hep-th/9611203.

[11] J. Polchinski, String Theory: Vols. I and II, (Cambridge University Press, Cambridge, 1998).

[12] C. Vafa, Nucl. Phys. B469 403 (1996), hep-th/9602022.

[13] S. Sethi, C. Vafa, and E. Witten, Nucl. Phys. B480 213 (1996), hep-th/9606122.

[14] K. Becker and M. Becker, Nucl. Phys. B477 155 (1996), hep-th/9605053.

[15] K. Dasgupta, G. Rajesh, and S. Sethi, JHEP 9908023 (1999), hep-th/9908088.

[16] B. R. Greene, K. Schalm, and G. Shiu, hep-th/0004103.

[17] J. Polchinski, Phys. Rev. Lett. 754724 (1995), hep-th/9510017.

[18] For excellent introductions see:

J. Polchinski, hep-th/9611050;

C. Bachas, hep-th/9806199. 
[19] P. Candelas and D. Raine, Nucl. Phys. B248 415 (1984).

[20] J. Polchinski and A. Strominger, Phys. Lett. B388 736 (1996), hep-th/9510227.

[21] J. Michelson, Nucl. Phys. B495 127 (1997), hep-th/9610151.

[22] T. Taylor and C. Vafa, Phys. Lett. B474 130 (2000), hep-th/9912152.

[23] P. Mayr, hep-th/0003198.

[24] J. Schwarz, Phys. Lett. B367 97 (103), hep-th/9510086;

S. de Alwis, Phys. Lett. B388 291 (1996), hep-th/9607011.

[25] I. Antoniadis et al., Phys. Lett. B436 257 (1998), hep-ph/9804398.

[26] I. Antoniadis and C. Bachas, Phys. Lett. B450 83 (1999), hep-th/9812093;

N. Arkani-Hamed et al., hep-th/9908146;

K. Dienes, E. Dudas, and T. Gherghetta, Nucl. Phys. B537 47 (1999), hep$\mathrm{ph} / 9806292$.

[27] A. Strominger, Nucl. Phys. B451 96 (1995), hep-th/9504090.

[28] P. Candelas, P. S. Green and T. Hubsch, Nucl. Phys. B330 49 (1990).

[29] Y. Hayakawa, alg-geom/9507016.

[30] P. Candelas, D. Diaconescu, B. Florea, D. R. Morrison and G. Rajesh, hepth/0009228.

[31] P. Mayr, Nucl. Phys. B494 489 (1997), hep-th/9610162.

[32] P. Mayr, private communication.

[33] F. Wilczek, Phys. Scripta T36, 281 (1991).

[34] C. Bachas and E. Kiritsis, Nucl. Phys. Proc. Suppl. B55, 194 (1997), hepth/9611205;

C. Bachas et al., Nucl. Phys. B509 33 (1998), hep-th/9707126.

[35] For excellent lectures, see:

E. Kiritsis, hep-th/9906018.

[36] I. Klebanov and A. Tseytlin, Nucl. Phys. B475 164 (1996), hep-th/9604089.

[37] N. Itzhaki et al., Phys. Rev. D58 046004 (1998), hep-th/9802042.

[38] J. Preskill et al., Mod. Phys. Lett. A6, 2353 (1991). 
[39] P. Kraus and F. Wilczek, Nucl. Phys. B433 403 (1995), gr-qc/9408003;

Nucl. Phys. B437 231 (1995), hep-th/9411219.

[40] E. Keski-Vakkuri and P. Kraus, Nucl. Phys. B491 249 (1997), hep-th/9610045.

[41] R. Bousso and J. Polchinski, hep-th/0004134. 